F O U N D T I O N O F C O M P T I N G A D D E C I S I O N S C I E N C E

Vol. 44

(2019)

No. 4

ISSN 0867-6356

sciendo

DOI: $10.2478 /$ fcds-2019-0019

e-ISSN 2300-3405

\title{
Mutants as Patches: Towards a formal approach to Mutation Testing
}

\author{
Tomasz Lewowski * $\quad$ Lech Madeyski ${ }^{\dagger}$
}

\begin{abstract}
Background: Mutation testing is a widely explored technique used to evaluate the quality of software tests, but little attention has been given to its mathematical foundations.

Aim: We provide a formal description of the core concepts in mutation testing, relations between them and conclusions that can be drawn from the presented model.

Method: We introduce concepts of mutant space and patch space, and refer to patch merging procedure from the patch theory. We explicitly present constraints, such as location-dependence, that affect mutation operators. We also present a way to use introduced formalism with traditional operators proposed in other papers.

Results: The proposed formalism allows to describe interactions between separate mutations using well-known abstract algebra notation.

Conclusion: The presented formalism may substantially decrease the number of tested weak mutants and increase the number of valuable ones, while giving tools to partially address the problem of equivalent mutants, particularly for higher-order mutation testing. However, additional empirical evaluation is still needed.
\end{abstract}

Keywords: mutation testing, fault-based testing, patches, equivalent mutants

\section{Introduction}

Mutation testing is a fault-based technique used for assessing test quality. In the process artificial changes are introduced to the source code. Such modified programscalled mutants - are then used to evaluate test suite. If any test case fails on modified

\footnotetext{
*Faculty of Computer Science and Management, Wroclaw University of Science and Technology, Wybrzeze Wyspianskiego 27, 50370 Wroclaw, ORCiD: 0000-0003-4897-1263, email: tomasz.lewowski@pwr.edu.pl

${ }^{\dagger}$ Faculty of Computer Science and Management, Wroclaw University of Science and Technology, Wybrzeze Wyspianskiego 27, 50370 Wroclaw, ORCiD: 0000-0003-3907-3357, email: lech.madeyski@pwr.edu.pl
} 
program, the mutant is said to be killed. A perfect test suite should kill all mutants that do not behave exactly like the original program. Despite its long history, starting at end of 70s [17, 12, 11, surprisingly little focus has been given to the theoretical foundation of mutation testing. While mutation testing is analyzed and developed, its basic assumptions still slip out of formal specification with rare exceptions [47, 5]. Hence, the aim of the article is to provide a minimal theoretical foundation for further work in mutation testing area.

This paper makes several contributions. The primary one is providing a distinct from existing ones and unequivocal definition of the basic concepts of mutation testing. Another contribution is an advanced but useful mathematical tool for further analysis, as well as preparation of domain-specific terms, definitions, constraints and goals that will be used in further research. It also sets the focus on mutation operators, which, are core elements of mutation testing. Consequences of imposing certain restrictions on operators are discussed in detail.

Some of the constraints introduced by the framework may seem too heavy for practical usage. While we believe that this is not the case, it is still too early to judge its usability. Even if some of the requirements are indeed too strict for general usage, they may turn out to be a reasonable choice for constrained, domain-specific languages (for example for logical expressions). The abstractions are introduced one by one, and in general each abstraction imposes additional constraints on mutation operators. Implementors and users are free to choose which level of abstraction is acceptable for them and utilize only the guarantees provided by these abstractions.

Our conjecture is that by utilizing the formal framework presented in this article it will be possible to substantially reduce both the testing effort related to useless HigherOrder Mutants (HOMs) and the number of equivalent mutants reported to the end user [33]. This can be done by introducing formal rules, structured as equations, and transforming the set of mutations into its minimal mathematical representation. This paper intentionally leaves some of the defined parameters, such as distance metric in Section 3 or location scheme in Section 6, without a specific instantiation. While we are aware that this might impact perceived expressiveness of presented model, at the same time we cannot present a single gold standard for this parametersbest values for given situations are still subject to further research. Therefore, not to lose generality, we abstract them and present them as subject to be instantiated during development of mutation testing tool (or possibly even at runtime). While we acknowledge that empirical evaluation of such framework would be beneficial, this is still subject for further research.

We structured the paper as follows: Section 2 introduces the closely related work (on which we will build upon) to the reader, Section 3 familiarizes the reader with the basic definitions used throughout the paper, Section 4 discusses the constraints on mutation testing we propose to impose, Section 5 presents the axioms, while Sections 6, 7 and 8 focus on our specific approach, discussion of the findings and conclusions. 


\section{Related work}

A general theory for fault-based testing (which is more general than mutation testing) was described by [37, 38. These papers introduce requirements which must be fulfilled in order for programs to be differentiable, discuss generation of reliable test sets and theoretically prove some theorems about them, like whether such sets can be generated for an arbitrary application.

There is a significant amount of research related to mutation testing in general. However, most of it focuses either on empirical analysis of certain features of mutation testing as a whole [44, 34, 4, analyzing features of operators [48, 49, 14, 19]), overcoming the equivalent mutant problem [33, or efficiency [16, 46, 53]). In the area of mutation operators, substantial amount of research was put into evaluating Statement Deletion (SDL) operator [9, 13. Overcoming equivalent mutant problem and efficiency are related to this article, as formal tools that will be provided may allow to detect some of the equivalent mutants a priori (before compilation). We also argue that analyzing features of mutation operators is the core activity to use provided mathematical framework.

A different formal approach to mutation testing — based on directed graphs — was given by [5]. Their framework focuses on representing program structure for mutation purposes and mutating on the level of program graph. While the model differs from presented in this paper, it is in fact complementary to it. That is because, since directed graphs are a useful mathematical concept that may be used for constraining program structure to provide addressing model, introduced in Section 6.3.

A general, behavior-based framework for mutation-based methods, not only for mutation testing, was presented by [47. We focus exclusively on mutation testing, mostly from structural point of view (as opposed to behavior-based approach presented by [47). Our main focus are mutation operators and their construction, therefore our set of definitions and terms differ. For example, test differentiator, a core concept introduced in [47, is only implicitly present in this paper in the form of an assertion in a test set. On the other hand, behavioral difference presented by 47. is close to being a special case of a more generic distance relations, which we leave abstract.

Finally, there is a number of real-world applications of abstract algebra that make it possible to solve problems in various areas of science and engineering. These include, amongst others, chemistry [6], physics [30], computer science [22] and cryptography [29]. Since the same mathematical concepts are used in such broad spectrum, we suppose that it may also prove being useful when applied to mutation testing.

\section{Definitions}

Let us introduce some basic terms and definitions on which we will build upon:

$S$ space of all possible text strings

$P$ space of all programs syntactically valid in any programming language 
$P_{\text {Lang }}$ space of all programs syntactically valid in programming language Lang

$p \in P_{\text {Lang }}$ the application that is being analysed

$P_{\text {Interface }}$ space of all programs in $P_{\text {Lang }}$ with the same public API as $p$

$m$ a mutant; in general, $m \in S$, but only $m \in P_{\text {Lang }}$ is usually useful, so only $m \in P_{\text {Lang }}$ will be analyzed

$O_{p} \quad$ space of all mutation operators

$O_{p c}$ a subspace of mutation operators which represent ones that are commutative ${ }^{1}$

op mutation operator; in general op $: P_{\text {Lang }} \rightarrow P_{\text {Lang }}$, but most operators represent op $: P_{\text {Interface }} \rightarrow P_{\text {Interface }}\left(P_{\text {Lang }} \rightarrow P_{\text {Lang }}\right.$ may be useful for analyzing effects of breaking changes in public APIs)

$t$ a test case, i.e. set of assertions that verify some property of a program and may either pass or fail

$T$ set of all possible pairs of (input, output) of a given program $p$ in form of test cases. This is a set that contains all possible scenarios of application execution, so it is not feasible to obtain it, but it is useful to set up the context for its relevant subsets.

$T_{0}$ set of all relevant test cases that can be written for a program, i.e., its formal specification (usually not present explicitly). This is mostly a concept that is useful for definitions and theorems. In most practical cases it will be approximated. Usually $T_{0}$ contains less constraints than $T$, simply because some application behaviors remain unspecified.

$T_{1}$ set of existing valid test cases, that may be executed at any mutant, i.e., ones that were already written; of course, $T_{1} \subseteq T_{0}$

test $(t, m)$ result of running a test case on mutant $m$. test $(t, m)=$ true if all assertions from $t$ are satisfied by $m$, false otherwise

$\operatorname{fail}\left(T_{x}, m_{x}\right)$ result of an operation that verifies whether a given set of tests $T_{x}$ detects mutant $m_{x}$, i.e. $\operatorname{fail}\left(T_{x}, m_{x}\right)=$ true $\Longleftrightarrow \exists t i n T_{x}: \operatorname{test}\left(t, m_{x}\right)=$ false. This is an operation used to introduce relation between a test set (such as $T_{0}, T_{1}$ or any of their subsets) and mutants. We decided to use this notation instead of $T_{x}\left(m_{x}\right)=$ true $\Longleftrightarrow \forall t \in T_{x}: t\left(m_{x}\right)=$ true to clearly separate data (test case) from evaluation procedure (fail)

$\operatorname{dist}\left(m_{x}, m_{y}\right)$ a number representing metric of similarity between $m_{x}$ and $m_{y}$. We intentionally do not define the meaning of similarity here, as various distance metrics may lead to various results. This is also a subject for further research, thus no specific metric can be given as the gold standard yet. The metric should

\footnotetext{
${ }^{1}$ operations $f_{1}$ and $f_{2}$ are commutative $\Longleftrightarrow \forall x f_{1}\left(f_{2}(x)\right)=f_{2}\left(f_{1}(x)\right)$
} 
fulfil mathematical requirements of a metric, i.e., $\operatorname{dist}(x, y) \geqslant 0 \wedge \operatorname{dist}(x, y)=$ $0 \Longleftrightarrow x=y \wedge \operatorname{dist}(x, y)=\operatorname{dist}(y, x) \wedge \operatorname{dist}(x, z) \leqslant \operatorname{dist}(x, y)+\operatorname{dist}(x, z)$ [3. Example metrics include edit distance [39] and number of affected program paths [28], but many others can be used as well, as long as they fulfill the aformentioned requirements.

\subsection{Example}

A simple example that we will expand during the whole paper is mutation testing of bubble sort algorithm.

Listing 1: Original program - BubbleSort

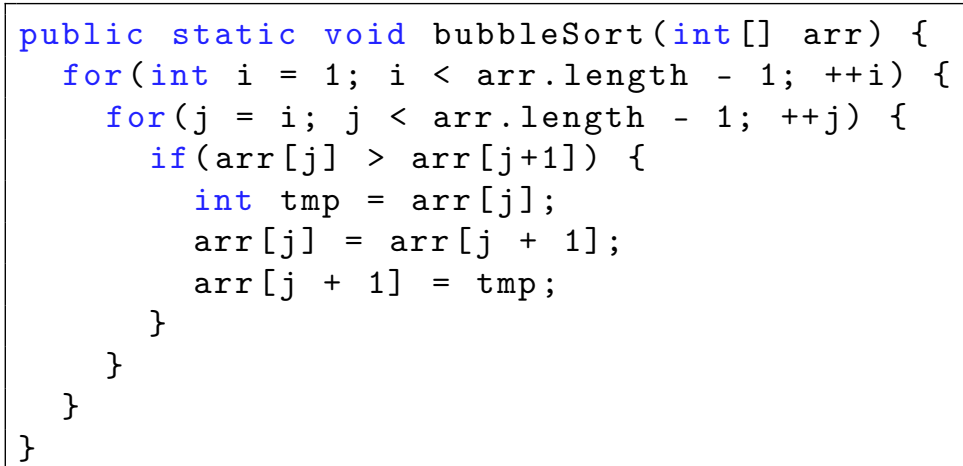

This program contains a bug-iteration starts from $i=1$ instead of $i=0$. As a result, $T$ and $T_{0}$ differ. Example test case that belongs to $T$ but not to $T_{0}$ is

$$
\text { bubbleSort }([2,1])=[2,1]
$$

Example test case that belongs to $T_{0}$ but not to $T$ is

$$
\text { bubbleSort }([2,1])=[1,2]
$$

Domains of both $T$ and $T_{0}$ are identical and they are both infinite sets-possible inputs include all arrays, possible outputs - all arrays arr that contain same elements as input array and $\forall \operatorname{arr}[i] \leq \operatorname{arr}[i+1]$.

The set of mutation operators that we will use will contain the following $(\mathrm{A} \leftrightarrow \mathrm{B}$ means an operator that applied to A transforms it to B and applied to B transforms it to A):

- comparison mutations: LEQ $\leftrightarrow$ GEQ, LEQ $\leftrightarrow$ LE, GEQ $\leftrightarrow$ GE

- arithmetic mutations: $+\leftrightarrow$ -

- delete statement

- change variable in statement into other one with matching type 
- change assignment to comparison

- change integer to 0

As a metric of similarity we will use edit distance, understood as number of characters that need to be added, deleted or modified to make both source codes identical.

\section{Constraints}

Only mutants that are interesting for language with static types are ones that are syntactically valid in the analyzed programming language. Syntactic issues are detected in the compile time, so they can be mitigated easily by the development team and do not require additional assistance from testing staff. This is not strictly true in case of dynamically typed languages, where syntactic error may not be detected until run-time. However, this kind of problems is usually fairly simple to detect using a variety of available tools, such as linters [23, 35, 7] and unit tests [52, 45]. Additionally, this type of errors is usually permanently visible as soon as code is executed (or imported into scope, depending on severity of the problem and language details), thus greatly reducing usefulness of $m \notin P_{\text {Lang }}$ - even though they may still be runnable it is immediately obvious that any test case that covers invalid statements would kill the mutant. Since coverage analysis is a tool that is easier to apply than mutation testing, we will not focus on mutants that violate language rules - simply because any problems that they may pose are already addressed by different tools. Therefore, the only mutants that are worth analyzed further are $m \in P_{\text {Lang }}$. It is also reasonable to restrict analysis further to $m \in P_{\text {Interface }}$ otherwise it is possible that one will not be able to execute same test suite against mutated application, which might be easily interpreted as killing a mutant (which renders these mutants useless, as will always be killed). Of course this is true only if mutated lines get executed (methods called, configurations used etc.), but if they do not, mutation testing is not the right tool - again, simple coverage analysis would suffice. Lines that are not executed may contain any code or no code at all and do not affect program output.

It is important to understand that - in sense of above definitions - program $p$ is just a special case of a mutant. Transforming between programs by applying mutation operators is no different than implementing a feature or fixing a defect. In fact, one may understand fixing a defect as applying some operation to original source code (with defect) to obtain modified source code (without defect). In typical scenarios this „operation” would be a change applied by a software developer. This change is then communicated to all parties interested in modified source code, often in form of a patch - machine-readable instruction for transforming between two structures of source code.

Changes applied by mutation operators are a subset of all possible changes. Therefore application of any mutation operator may be understood as application of a patch (modification) over a program source code. Thus the only two constraints are discussed: 
1. its output must always be a syntactically valid program in a given programming language,

2. the public API specification (most notably function signatures) must remain unchanged. This should not be understood as preservation of implicit contracts (e.g. HTTP or SOAP ones), but only as minimal sanity (ability to run local instance of application).

A subtle, yet important detail is that $T$ and $T_{0}$ are not necessarily related. $T$ takes inputs and outputs from program $p$ as-is, which means that for $T$ and all its outputs of $p$ are meaningful. $T_{0}$ on the other hand may discard some irrelevant outputs (e.g. random number requests, system time accesses etc.). An implicit effect is that $T$ may contain elements, which are not part of $T_{0}$, i.e., in general $T_{0} \nsubseteq T$ (acceptable outputs - ones in $T_{0}$ - may include various versions of log messages, message ordering, number of retries etc., but $T$ contains only single output for each input) and assumption that $T \subseteq T_{0}$ is equivalent to assuming that program does not contain defects (all input-output pairs are acceptable from the perspective of formal specification). However, for simplicity, further on it is assumed that test cases picked by the programmer are picked from the set of valid test cases, as, according to our current knowledge, an automatic tool has little chance of detecting non-trivial invalid test cases without understanding of program specification (program understanding is another wide area or research, but it is currently out of scope of mutation testing), which means that for further discussion we will assume $T_{0} \approx T$-i.e., we will treat $p$ as an oracle and set of all its input-output pairs as the formal specification of $p$. This assumption is not entirely obvious, but reasonably intuitive - if we want to verify equivalence according to specification, but we have no specification, we verify for all possible input sequences.

\section{Axioms}

Mutation testing makes two assumptions about the original program:

1. $\operatorname{fail}\left(T_{1}, p\right)=$ false, i.e., all tests pass on the original program,

2. $\operatorname{fail}\left(T_{0}, p\right)=$ false, i.e., original program is the actual specification

The second assumption might seem strange, since it might be interpreted that the tested program is compliant to its specification. This is not a correct interpretationthe program does not have to be compliant to its specification (whatever its specification is - often it is not even known), but during mutation testing process we search for programs that exhibit same behavior as $P$, regardless if it is valid from business perspective. Therefore, we can assume that for generated mutants $T_{0}$ is the important subset of $P$ behavior (whole behavior is defined as $T$ and may contain also outputs and actions irrelevant to business, like random number acquisition, time clock checks, logging etc.).

Since a mutation tester aims to create mutants that help to discover missing test cases, one will aim to create mutants $m$ that fulfill three requirements: 
1. $\operatorname{fail}\left(T_{0}, m\right)=$ true - the mutant is not equivalent (according to formal specification), i.e., there does exist a test case, $t_{f}$ that fulfills specification $\left(t_{f} \in T_{0}\right)$ and fails on mutated program. Mutants for which $f a i l\left(T_{0}, m\right)=$ false are called equivalent mutants,

2. $\operatorname{fail}\left(T_{1}, m\right)=$ false - the mutant is not detectable with current test set, i.e., $t_{f} \notin T_{1}-t_{f}$ is not part of existing executable test suite,

3. $m \approx p$-the mutant is similar to the original program $p$. This means that there exists some similarity metric, which indicates that mutant is close to the original program and its value is relatively small. Exact meaning of small may depend on multiple factors, such as planned effort for mutation testing.

These three requirements may lead to a formal definition of mutation testing:

Definition 1 Mutation testing is a process of evaluating test set $T_{1}$ by finding a set $M_{\varepsilon}$ of programs such that for given $\varepsilon$ and $p: m \in P_{\text {Interface }}$ and fail $\left(T_{0}, m\right)=\operatorname{true}$ and $\operatorname{fail}\left(T_{1}, m\right)=$ false and $\operatorname{dist}(p, m)<\varepsilon \Longleftrightarrow m \in M_{\varepsilon}$

For instance, for example described in Subsection 3.1 one such mutant appear when we apply „change integer to 0 ” operator to $i=1$ resulting in $i=0$. This will generate a mutant $m$ that definitely cannot be detected by $T_{1}$ because of our assumption that $T_{1} \subset T_{0}$ and $m$ is actually the correct implementation. Other mutant in $M_{\varepsilon}$ will be obtained by changing $\operatorname{arr}[j]>\operatorname{arr}[j+1]$ into $\operatorname{arr}[j] \geqslant \operatorname{arr}[j+1]$ - this one is equivalent. Depending on the exact contents of $T_{1}, M_{\varepsilon}$ may contain also other mutants.

When defined as such, every mutation testing algorithm shall have at least three parameters - fail operation, dist operation and $\epsilon$ parameter ${ }^{2}$

A key factor for finding programs satisfying Definition 1 is to define proper way of exploring the search space. A typical way of achieving it is to apply mutation operators to certain locations in program. When applying multiple mutations, an important factor is whether mutations are applied at once or as a sequence of changes. Applying a sequence of changes increases risk of conflicts, such as reversing own change, while using a set of changes allows the mutation tester to apply techniques developed for patch theory 2] - detect conflicts and resolve them (possibly by rejecting some of conflicting changes). Using patch theory is possible for both sets and sequences of changes, but may require more development effort and additional bookkeeping when implemented in the context of sequences of changes.

In the subject literature [21, 40] mutants with multiple changes are called higher order mutants (HOMs $\widehat{\gamma}^{3}$ contrary to mutants with only a single application of one mutation operator, called first order mutants (FOMs) [18]. This classification may give a false impression that FOMs are, generally speaking, more similar to original program than HOMs. This impression is caused by the underlying assumption (often not explicitly expressed) that every mutation (application of mutation operator)

\footnotetext{
${ }^{2}$ Theoretically it is sufficient to merge dist and $\epsilon$ into a single operation, but we will not apply this step, as it removes orthogonality from the solution.

${ }^{3}$ Strongly subsuming higher order mutants are of particular interest [4].
} 
introduces a similar level of difference. This assumption can be formally expressed as:

$$
\begin{aligned}
& \forall \text { op }_{1} \in O_{p}, \forall o p_{2} \in O_{p}, \forall p \in P_{\text {Lang }}, \forall T_{1} \subseteq T_{0}: \\
& \operatorname{dist}\left(p, o p_{1}(p)\right)=\operatorname{dist}\left(p, o p_{2}(p)\right)
\end{aligned}
$$

Considering huge number of proposed mutation operators [10, it seems very unlikely for such assumption to hold for any dist function that is not defined in terms of $o p$ (and according to definition in Section 3 dist cannot be defined in terms of $o p$, as arguments of dist are programs, not initial program and sequence of mutations). There are no widely accepted constraints regarding what can and what cannot be a mutation operator [25, 31, 48] - thus any general theory would need to be able to handle arbitrary operators, including ones that remove whole implementation or rewrite it. Second, even the same mutation operator applied in different places may result in various distances. Consider the two code snippets on Listings 2 and 3 .

Listing 2: An obvious defect introduced by a mutation

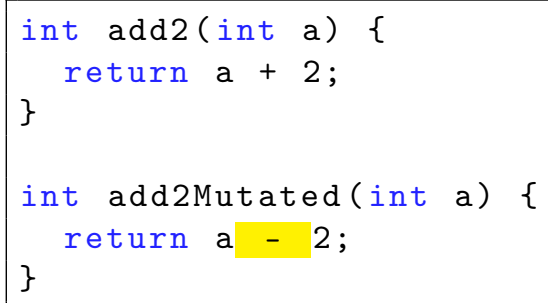

Listing 3: A change introduced by mutation - it is not obvious whether this is a defect

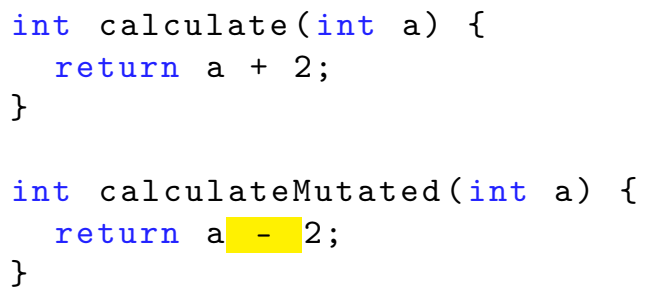

From the syntax perspective, both are identical. However, the first function (add2Mutated) does something contrary to the name, which - to a minimally competent developer - indicates a place where a defect is likely to be found. The other one calculateMutated has meaningless name, thus it is not clearly visible whether the implementation is correct or not. While usually relying on name to provide semantics is troublesome (especially in software that might operate on AST or bytecode level), some other areas of software engineering, such as Elemental Design Patterns [50] or issue-commit link mining [51] have successfully adopted such approach. Using this additional semantic information available in the source code is reasonable, since it 
represents a significant part of developers' knowledge. It is also possible, as this information is present in source code (thus can be used directly in static analysis), and usually can be retrieved at runtime from built software (e.g. using symbol maps).

Similar examples are often used during software development lessons - that verbose names mean much to people. Taking into account The Competent Programmer Hypothesis, the second defect is much more likely to appear, so its distance from the original program should be smaller.

The division between FOMs and HOMs is largely based on the assumption that FOMs are much more similar to original program than HOMs. While this is intuitively true, this definitely does not mean that all defects represented by FOMs are equally likely to be introduced in code [42] by developers, and, more importantly, it does not mean that this difference somehow scales with the order of mutant. For example, consider the following original program in Listing 4

Listing 4: Original program for different HOM distance

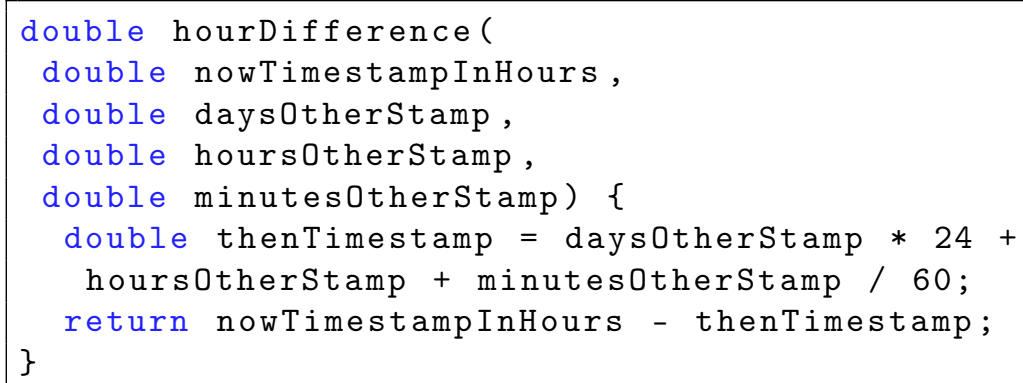

And possible two Second Order Mutants in Listing 5

Listing 5: Two example Second Order Mutants

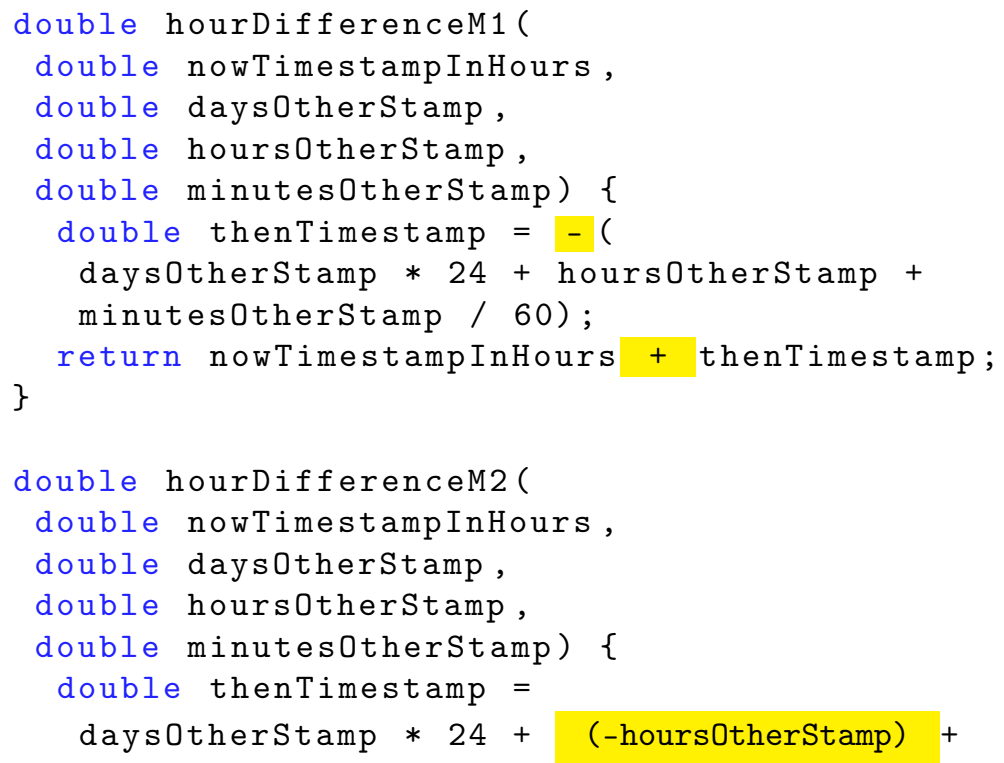




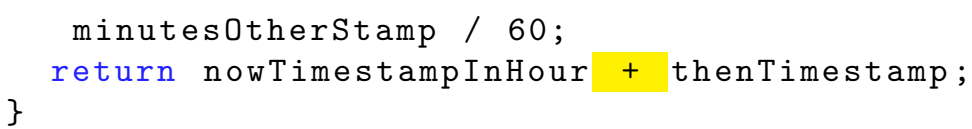

While neither of them appeals to a software developer, they present an interesting situation - the first mutant is equivalent, while the second one is different. While both differ syntactically from the original program by the same number of changed AST nodes (2), it would be counter-intuitive to say that the distance is same in both cases. This example covers only two mutation operators, both arithmetic. In realworld applications, there are dozens of mutation operators of all kinds (arithmetic, logic, polymorphic, container-based, semantic etc.) - one cannot simply assume that they all have similar impact.

Another important point in mutation testing theory is the structure of $P$ (space of all possible programs) which was not yet thoroughly studied, and this article aims to scratch the surface of this problem. The widest approach to defining the space itself is to define it as set of all possible character strings. This is the upper bound, but this already gives an important hint, namely that the mutant space is - at mostcountable.

Next reasonable attempt to make the space smaller is to accept only those strings, which are syntactically valid programs in selected language. This creates the entire set of mutant spaces, $P_{\text {Lang }}$ - one for each programming language- but of all of them, only a single one is valid at any given time (assuming only single language mutations are allowed, which is a reasonable constraint). Creating mutants that are not valid programs makes little sense, as they are detected before the program even starts, during compilation phase (as discussed in Section 4). Next change in the mutant space may be done basing on the public API - if the mutation changes public API (function names and parameters), then programs will either not compile, or crash at runtime-so these mutations also are useless. The remaining space, $P_{\text {Interface }}$, remains infinite, but countable.

We define a concept of neighborhood:

Definition $2 \forall m_{1} \in P_{\text {Interface }} \forall m_{2} \in P_{\text {Interface }}:$ neighbor $\left(m_{1}, m_{2}\right)=$ true $\Longleftrightarrow$ $\exists$ op $\in O_{p}: o p\left(m_{1}\right)=m_{2}$, where $O_{p}$ is a set of mutation operators allowed to be used in current algorithm

This means that $m_{1}$ is a neighbor of $m_{2}$ if and only if there is a direct transition between them, i.e., there is an operator, that - when applied exactly once - performs transformation from $m_{1}$ into $m_{2}$. While this might not be intuitive, neighborhood relation is not always commutative. This is due to the fact that set of used mutation operators - in most current mutation testing tools - is configurable by user, so it is not guaranteed to be invertible (i.e., for given operator $o p_{1}$ it is not guaranteed that there exists an operator $o p_{2}$ such that $\forall p \in P_{\text {Interface }}, o p_{2}\left(o p_{1}(p)\right)=p$. If such an operator exists in $O_{p}$, then we will call $o p_{2}$ an operator inverse to $o p_{1}$. If $o p_{2}$ is inverse to $o p_{1}$ and $o p_{1}$ is inverse to $o p_{2}$, they are reverse operators, and they are both reversible in $O_{p}$. 
Definition 3 If for given operator op $p_{1}$ from set of mutation operators $O_{p}, \forall p \in$ $P_{\text {Interface }}, \exists o p_{2} \in O_{p}: o p_{2}\left(o p_{1}(p)\right)=p$ then we will call $o p_{1}$ invertible and $o p_{2}$ is an operator inverse to op 1

Definition 4 If every operator in $O_{p}$ is invertible, $O_{p}$ is an invertible operator set

We define that mutant $m_{1}$ is reachable from mutant $m_{2}$ if there exists a sequence of mutation operators (from defined set) which transforms mutant $m 1$ into $m_{2}$.

Definition $5 \forall m_{1} \in P_{\text {Interface }}, m_{2} \in P_{\text {Interface }}$ : reachable $\left(m_{1}, m_{2}\right)=$ true $\Longleftrightarrow \exists o p_{1}, o p_{2}, \ldots, o p_{n}: o p_{1} \ldots o p_{n} \in O_{p}:$ $o p_{n}\left(\ldots\left(o p_{2}\left(o p_{1}\left(m_{1}\right)\right) \ldots\right)\right)=m_{2}$ where $O_{p}$ is the set of mutation operators used in the algorithm.

\subsection{Invertibility imposed on mutation operators}

If every mutation operator in the used set is invertible, $P_{\text {Interface }}$ can be divided into clusters, $P_{C}$ - every element of a cluster is reachable from other element in the cluster. Cluster might be infinite (depending on mutation operators), but for practical reasons the search should be constrained, e.g., by some heuristic on dist, usually just maximum number of mutations or maximum mutation time. Only a single cluster can be analyzed when using mutation testing, because the starting point for mutation ( $\operatorname{program} p$ ) is fixed, and single point can only belong to one cluster.

Definition $6 \forall m_{1} \in P_{C}, m_{2} \in P_{C}$ : reachable $\left(m_{1}, m_{2}\right) \Longleftrightarrow \operatorname{reachable}\left(m_{2}, m_{1}\right)$

Definition $7 \forall m_{1} \in P_{C}, m_{2} \in P$ : reachable $\left(m_{1}, m_{2}\right) \Longleftrightarrow m_{2} \in P_{C}$

\subsubsection{Example}

If we decide to use only invertible operators in our example, we need to inspect the original list. It contains comparison mutations, arithmetic ones, statement deletion, chariable change and assignment to comparison change. Now, if we insist on leaving only invertible ones we definitely need to reject statement deletion (to invert it we would need to create an opeartor that inserts arbitrary code). Both comparison operators and arithmentic ones fulfill our requirements. Changing variable into other one may (but does not have to) match our requirements, depending on detailed implementation - for simplicity, we will reject it now. Changing assignment to comparison is not invertible according to our set of operators, but it is relatively simple to make it invertible - add a reverse operator, change of comparison to assignment. Note that this operator cannot be applied anywhere in the original program, but nevertheless needs to be part of our operator suite. 


\subsection{Mutation testing as a problem of reachability}

In Section 5 we have defined the operational goal of mutation tester as finding other programs which are different than the original (but similar to it), and the difference is not detectable by existing test set (more widely, these are used to evaluate the test set and, possibly, improve it). Here we provide an additional constraint - the other program must be reachable from the original one by using a series (or set) of applications of chosen mutation operators. This requirement is somewhat similar to the one regarding similarity, but is much better defined (for given set of mutation operators), and easier to apply. It also does not cover some parts of the original requirement, since it is possible to create a program substantially different from original which will pass all tests. Up to now we did not impose any constraints on the nature of mutation operators - those have a critical impact on whole process of mutation testing and types of mutants that may be evaluated.

Theorem $1 \forall m_{1} \in P_{\text {Interface }} \forall m_{2} \in P_{\text {Interface }}, \forall m_{3} \in P_{\text {Interface }}$ : reachable $\left(m_{1}, m_{2}\right) \wedge$ reachable $\left(m_{2}, m_{3}\right) \Rightarrow$ reachable $\left(m_{1}, m_{3}\right)$

Proof: Let $m_{1}, m_{2}, m_{3} \in P_{\text {Interface }}, o p_{1}$ be a mutation operator or a chain of $n_{1}$ mutation operators such that $o p_{1}\left(m_{1}\right)=m_{2}$ and $o p_{2}$ be a chain of $n_{2}$ mutation operators such that $o p_{2}\left(m_{2}\right)=m_{3}$. Then, by definition, reachable $\left(m_{1}, m_{2}\right)=$ true and reachable $\left(m_{2}, m_{3}\right)=$ true. Since $o_{2}\left(m_{2}\right)=o_{2}\left(o p_{1}\left(m_{1}\right)\right)=m_{3}$, reachable $\left(m_{1}, m_{3}\right)=$ true, and the mutation order (number of applications of mutation operators) is at most $n_{1}+n_{2}$.

Reachability is always associative. Reachability is commutative if, and only if, mutation operator set is reversible.

\section{Relations between mutation operators}

While the general approach to the mutant space gives some insight into the structure of mutation testing, it may not be sufficient for analyzing relations between mutants and properties that might be preserved. For this reason we now introduce a new structure, that might be useful for higher order mutation testing.

\subsection{Patch space}

In the context of mutation testing, program $p$ is a special one - it is a starting point for mutation testing process. We are going to transform $P_{\text {Interface }}$-space of all reasonable mutants - into space of patches $X_{p}$. A patch is a function that can be applied to the original program giving a mutant as a result. We are going to call invocation of patch on a program a patch application, and use syntax: $x(p)$. Patch application is defined only for the analyzed application $p_{0}$ and is related to transformation $P_{\text {Interface }} \Rightarrow X_{p}: \forall m \in P_{\text {Interface }}, x \in X_{p}: m \Rightarrow x \Longleftrightarrow x\left(p_{0}\right)=m$. Informally 
speaking, patch application may be understood in the way UNIX tools do it (although structural requirements imposed on the patch itself differ). merge is defined as composing patch functions, i.e., merge $\left(x_{1}, x_{2}\right)$ is a higher-order function [15] - a function which returns a function - and merge $\left(x_{1}, x_{2}\right)(p)=x_{1}\left(x_{2}(p)\right)$.

An important outcome from this analysis is that - since merge is associative$X_{p}$ creates a semigroup. In fact, it forms a monoid. Proof. Transformation of the original program, $p_{0}$ to $X_{p}$ yields a null patch $\left(x_{n u l l}(p)=p\right)$. Since this null patch applied to any program returns it, merge $\left(x, x_{n u l l}\right)=x$ and merge $\left(x_{n u l l}, x\right)=x$. This means that $x_{n u l l}$ is an identity element in $X_{p}$, and $X_{p}$ with merge operation form a monoid [27], $\left(X_{p}, x_{n u l l}\right.$, merge $)$ - from now on we will refer to them as $\left(X_{p}, \mathbb{1}, \cdot\right)$. \#.

This reasoning holds under an assumption that any two patches may be merged and will produce a valid patch. Note that this requirement is not easy to guarantee, and has received attention in the industry mainstream. For example, well-known version control systems like Git or Mercurial do not allow arbitrary patches to be merged, and there is a sophisticated patch theory describing required relationships between patches [20, implemented in a less known tool, Darcs. While patches in Unified Diff Format [1] (typical UNIX format) may be used in the implementation of the above system, it would be required to specify a conflict resolution algorithm or accept the fact that merge would be only a partial function (of course, one can also try to mitigate it by not allowing conflicting mutations to appear in $X_{p}$, but that might require manual inspection). This is due to fact that typical patches were invented to provide a transition between two arbitrary states of the code. However, in the mutation testing analysis we do not require transition to an arbitrary program, since there is a requirement regarding interesting mutants - they have to be similar to the original program. Nevertheless, patches can be used as the implementation of mutation operators.

\subsection{Mutation operators-requirements}

It is worth noting that - in above discussion - mutation operators were treated as unary functions. While inference basing on this assumption was valid, it is not very intuitive during discussion about the nature of the merge operation and requirements it imposes on the mutation operators.

Definition 8 An addressing $L$ is a way of uniquely identifying each statement of a program, which is preserved during the process of mutation testing.

We do not propose any gold standard for addressing - various techniques can be used, starting from simple statement counter, up to complex relationship dependencies. As of now, we cannot reliably predict which of those will be most useful for mutation testing systems - for future discussion, we simply assume that such an addressing exists.

From now on we will use a concept of mutator op - a pair $(f, l)$, where $f$ corresponds to a mutation function (the ,proper" mutation operator - one that is usually referred to in literature) and $l$ corresponds to a location, where $f$ is applied. For 
clarity for each program $p$ we will define $o p(p)=x=f(l, p)$, and $O_{\text {all }}$ is a set of all mutators that can be represented in this way for program $p$. Let also $O_{u}$ be a set of used mutators (of course $O_{u} \subset O_{\text {all }}$ ). Mutator is conceptually equivalent to a patch, but we use a separate term to denote its different origin.

\subsection{Example}

Continuing the bubble sort example, we provide a simple example addressing: statement/entity number. This means that inti would be statement 1 , inti $=0$ - statement $2, j$ - statement 3, arr.length - statement 4, arr.length - 1 - statement 5, $j<$ arr.length -1 - statement 6 etc. This means that we made the number of statements/entities rigid, and our "delete statement" operator will actually become "replace with empty statement".

Requirement 1 In order for $\left(X_{p}, \mathbb{1}, \cdot\right)$ to form a monoid, every code location $l$ must be resolvable for every sequence of preceding mutators, i.e., let $D_{f}$ be domain of $f_{x}\left(l_{x}, p_{x}\right)$. Then $\forall\left(f_{x}, l_{x}\right) \forall o p_{1}, o p_{2}, \ldots, o p_{n} \in O_{\text {all }}:\left(o p_{1} \cdot o p_{2} \cdot \ldots \cdot o p_{n}\right)\left(p_{0}\right) \in D_{f}$

This means that no matter what mutations were performed earlier, it must be possible to unambiguously resolve $l_{x}$. This is equivalent to a statement that there is some structure in $p_{0}$ which must be preserved during every mutation - the structure is represented by $l_{x}$ addressing. The nature of this structure does not need to be defined globally for every operator set - it can be defined per $O_{u}$. One can decide about the structure which has to be preserved - for example if the structure is the line or statement number $-l_{x}$ represents a range of affected statements. Note that such structure means that operators which add or remove lines/statements are not allowed in this specific model (empty lines are allowed by addressing and/or empty addresses are left between statements), but can be enabled by using a different addressing scheme.

The requirement above leads also to a more subtle constraint imposed on the mutation operators. As we have already stated, they need to preserve some predefined structure. However, when designing mutation operator one cannot assume that during execution it will encounter same structure as in the original program - the nature of mutation operators becomes a more general function, that may need to handle several types of inputs. To illustrate this fact with example, let us consider two mutation operators defined in a more typical way $-M_{1}$ transforming each and operator it encounters into or and $M_{2}$, transforming and operator into xor. Now let us consider the code in Listing 6

Listing 6: Original function

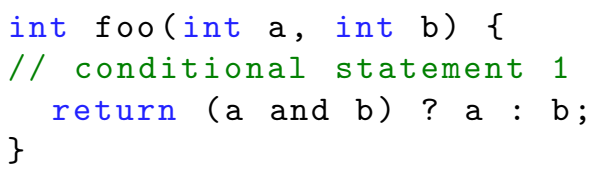


After applying only $M_{1}$ in conditional statement 1 we would get the code presented in Listing 7 .

Listing 7: Function after $M_{1}$ mutation

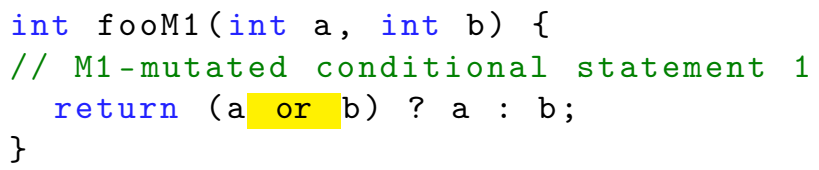

However, $M_{2}$ was also applicable to original conditional statement 1 , and would give in result code in Listing 8

Listing 8: Function after $M_{2}$ mutation

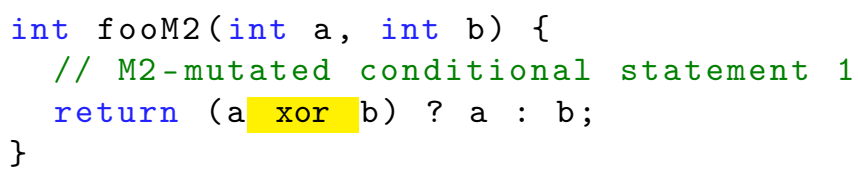

Both $M_{1}$ and $M_{2}$ were applicable to function foo, but it is clear that neither $M_{1}$ is applicable to foom2 nor $M_{2}$ to fooM1. This breaks Requirement 1, and as such, is not allowed. What is even more troublesome, even $M_{1}$ is not applicable to foom1 and $M_{2}$ is not applicable to fooM2 because they were explicitly defined as ,transfrom $<<$ and $>>$ to ...", but there is no $<<$ and $>>$ any more.

Yet operators in this form are very common and reusing them and knowledge related to them is an important issue. It arised from the fact of moving the logic of merge operation to the operators. There are three ways to solve the problem when there is given mutator $o p=\left(f, l_{x}\right)$ and operation of $f$ is defined only for a specific subset of structures that might be present in location $l_{x}$ :

1. If $f$ is not defined for structure in $l_{x}$, leave the structure as-was (i.e., for conflicting $\left.\left(f_{1}, l_{x}\right): f\left(l_{x}, f_{1}\left(l_{x}, p_{0}\right)\right)=f_{1}\left(l_{x}, p_{0}\right)\right)$.

2. Regardless of the structure that is in $l_{x}$, replace conflicting structure with the result of the original application (i.e., for conflicting $\left(f_{1}, l_{x}\right): f\left(l_{x}, f_{1}\left(l_{x}, p_{0}\right)\right)=$ $\left.f\left(l_{x}, p_{0}\right)\right)$.

3. Modify $f$ to extend its domain. This might be done for example by merging several typical mutation operators into one. For example, we could take operator $M_{1}$ defined above and add two other rules to it - for instance "replace or to xor and xor to and" — which would make the resultant operator — let us call it $M_{3}$ - applicable to all the three: foo, foom1 and foom2. It is also worth noting that $M_{3}\left(M_{3}(f \circ o)\right)=M_{2}(f \circ o)$, which means that they are not both simultaneously in the base of a monoid.

These solutions are used in virtually any version control system around the world in case of conflicting patches (sometimes they are called merge strategies). In mutation testing there is a need to perform every merge automatically, so during application 
operator (or some controlling entity) must be "aware" of what do to during conflict. Of course, this does not discard the possibility of automated full-program analysis for best resolution.

Note that while the first two options can be applied in presence of any patches preserving $l_{x}$ addressing, the third one cannot and generally might yield a partial function. This is not an acceptable constraint in the general patch theory, as applied in version control systems (that is when manual resolution is needed), but it is acceptable for the purposes of mutation testing. All three strategies boil down to transforming a partial function into a total one.

\subsection{Patch theory}

During studies related to patch theory [20, 2, 36] it was shown that if the system allows arbitrary patches, it can be represented as an inverse semigroup. However, during mutation testing only specific set of patches can be applied, and they do not need to keep this property (i.e., that for $\forall x \in X_{p} \exists x^{-1}: x \cdot x^{-1} \cdot x=x \wedge x^{-1} \cdot x \cdot x^{-1}=x^{-1}$ ). In the further discussion we will limit our concern to these subsets of mutation operators that form inverse semigroups, $O_{i s}$ - namely ones that are invertible in the meaning of Definition 3. Since it was proven earlier that every valid set of mutation operators forms a monoid, $O_{i s}$ forms an inverse monoid (but not necessarily a group).

\subsection{Patch independence}

We are going to define two patches, $x_{1}=\left(f_{1}, l_{1}\right), x_{2}=\left(f_{2}, l_{2}\right) \in X_{p}$ as independent $\Longleftrightarrow l_{1} \cap l_{2}=\varnothing$.

Independence is an important feature, because independent patches are trivially commutative (see 20] for definitions of commutativity in the context of patch theory), i.e., $x_{1}\left(x_{2}(p)\right)=x_{2}\left(x_{1}(p)\right)$. Note that there is no restriction as to what is represented by $l_{x}$ - one example was unique number of affected statement, which is relatively generic. Another example - arguably more sophisticated - would be a set of all nodes in the program flow graph which might be affected by the result obtained in the node. This representation might be more interesting, as may allow to significantly (depending on the application) decrease number of necessary higher-order mutants. This is because if two independent patches, $x_{1}$ and $x_{2}$ cause respectively $T_{f 1}$ and $T_{f 2}$ test sets to fail, mutant obtained by applying a merged patch, $x_{1} \cdot x_{2}$ will simply cause to fail a set of test cases $T_{f 1} \cup T_{f 2}$. Such conclusions are possible regardless of the actual details of structure of both patches, original program and content of test cases. This can be formalized in the following way:

Theorem $2 \forall x_{1}, x_{2} T_{f 1}:\left\{t \in T_{1}: \operatorname{test}\left(t, x_{1}(p)\right)=\right.$ fail $\}, T_{f 2}:\left\{t \in T_{1}: \operatorname{test}\left(t, x_{2}(p)\right)=\right.$ false $\}: x_{2}\left(x_{1}(p)\right)=x_{1}\left(x_{2}(p)\right) \Longrightarrow T_{f 12}=T_{f 1} \cup T_{f 2}$

Proof. Let $N_{1}$ be the set of nodes traversed during evaluation of $x_{1}$ and $N_{2}$ be the set of nodes traversed during evaluation of $x_{2}$. Since the assumption is that 
$N_{1} \cap N_{2}=\varnothing, T_{f 1} \cap T_{f 2}=\varnothing$. If it was not the case, it would mean that there is a test case that fails and runs through both $N_{1}$ and $N_{2}$. However, this is not possible, because in such case there would be a node $n_{1} \in N_{1}$ that would affect $n_{2} \in N_{2}$, which is not possible according to initial assumption. Therefore, since node sets are independent and failed test sets are independent, no interaction is possible between these two mutations, thus failed test case set must be a sum of failed test case sets for each of them. \#. It is important to note that this feature is specific to a given addressing - one that includes whole dependent subgraph. It will not hold if the addressing simply uses line or statement number.

The nature of patch independence, as understood in this paper, is neither syntactic nor semantic itself - it depends solely on $L$ addressing model. Change of addressing model may require a change of approach towards merging strategy - if the approach with dependency graph is used, there is little sense in using the first or second described general strategy. Instead, the patch should be usually applied to the code, partially overriding results of previous patches.

Definition 9 A Mutation Testing System is a tuple $\left(O_{p}, L\right)$ where $O_{p}$ is a set of mutation operators that can be applied in the system and $L$ represents used addressing model.

Definition 10 n-th order mutant is a mutant that can be represented at minimum by $n$ locations of application of commutative patches from the base.

Let us consider a simple example of when formal approach may yield useful results. Let our operator set contains two operators: negate (which negates a boolean) $N_{l}$ and logical (which transforms each and to or and or to and) $L_{l}$. In both cases lowercase $l$ represents location where operator shall be applied. Original function is simple:

Listing 9: Original function

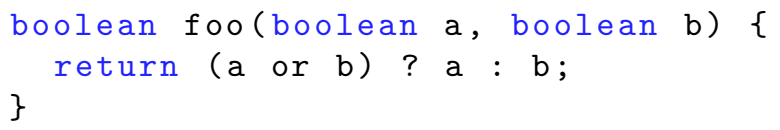

after first application of logical operator we get:

Listing 10: Function after applying logical operator

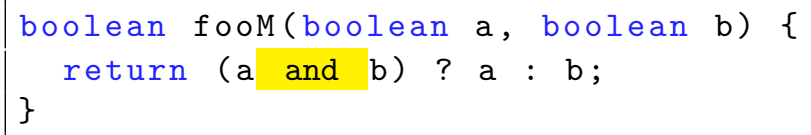

after first application of negate operator to result we get:

Listing 11: Function after applying logical operator and negate once

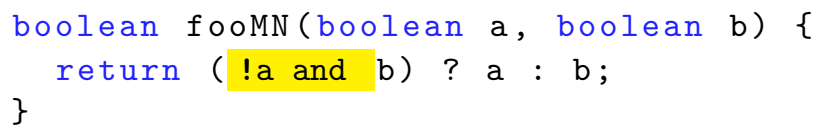


and after second application of negate we get:

Listing 12: Function after applying logical operator and negate twice

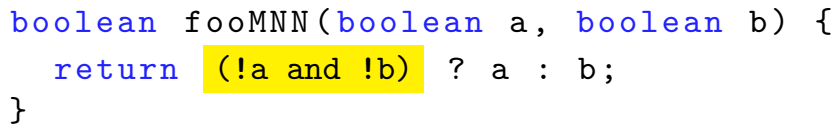

after third application of negate we get:

Listing 13: Function after applying logical operator and negate three times

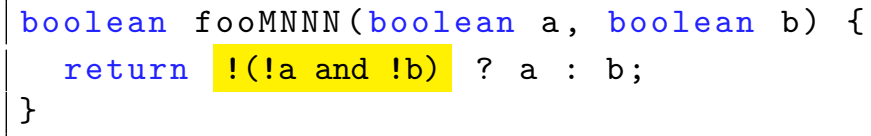

which is an equivalent mutant - one of the possible equivalent mutants from this set of operators. Let us describe it in notation used in the paper: $M_{2-3} \cdot N_{2} \cdot N_{3} \cdot N_{1}=\mathbb{1}$. Since $\cdot$ operation is associative, we can write it as $M_{2-3} \cdot\left(N_{2} \cdot N_{3} \cdot N_{1}\right)=\mathbb{1}$. Now there are a few steps to be taken:

1. $N_{1}, N_{2}$ and $N_{3}$ are commutative

2. $N_{1} \cdot N_{2} \cdot N_{3}=M_{2-3}$

3. $M_{x} \cdot M_{x}=\mathbb{1}$, i.e., $M_{x}=M_{x}^{-1}$

In the end it was provable that an equivalent mutant will be obtained. This was of course a simple example, but it demonstrates the power of the notation working in concert with specific features of patches and relations between mutation operators (and, consequently, resultant patches). When mutation operators are expressed as functions, such relations may be expressed as fact:4 4 and stored in some knowledge database. This example also demonstrates risks of using operators which are not part of the monoid base - when operators are not part of base, the risk of achieving the same program by different series of mutations arises.

\section{Discussion}

In this paper a formal system that can be used for inference about mutant features before their creation was presented. This can be perceived as an extension of papers by [37, 38] - all theorems from these articles remain valid, but we have provided a more detailed description of a model tailored specifically for mutation testing. Structure constraint required in the presented model may be provided by research published by [5], extending its primary effect scope.

Presented theory - when implemented - may have significant impact on how to deal with the problem of equivalent mutants. Current approaches concentrate rather

\footnotetext{
${ }^{4}$ Logical sentences that are always true.
} 
on post-creation detection whether mutant is equivalent to the existing program 43 , 24, 26]. This is an approach different than presented in this article-we have presented a notation that might be used to avoid creating equivalent mutants in the first place, as well as avoiding to create mutants for which it is already certain that they would be killed. Such approach may minimize both testing and mutation effort (which might be significant, especially if compilation is required for analysis) and act as a useful filter, leaving dynamic analysis only for mutants, which cannot be inferred. Additionally, notation presented in this article may reduce test effort required for mutants obtained by different operators (thus with different source, AST and bytecode), but behaving the same way - this is a unique feature coming from defining proper relations between mutation operators, such as in Listings 9. 13 . Three similar concepts related to introducing changes were used, so it may be useful to precisely recapitulate their scope:

- Mutation operator is a function that can be applied to a specific location in code. Mutation operator can be applied multiple times in the same location and may yield different results for each application. Operators are defined by user or mutation testing application.

- Patch is a single result of mutation operator application. Patches can be merged to form other patches. Patches form at least a monoid, but in special cases may also form a group.

It is also critical to understand that, even though the theory goes into details of operators forming a group, it is not strictly required to use only those. Instead, the developer (or even user) of the system should make an informed decision whether to use a restricted set of operators with additional benefits (e.g., decreased probability of equivalence) or an extended set with additional risks. In practice we do not expect users to opt-in for all possible features, but to understand the trade-off and pick a set of operators that best suits their needs.

Additionally, in this paper a minimal set of entities required for a mutation testing algorithm were defined. These include:

fail a function determining whether a mutant is killed by a given test suite

dist distance metric between mutants

$\varepsilon$ maximum distance in the search space which will be analyzed for interesting mutants

$O_{p}$ a set of mutation operators

$L$ addressing mechanism for mutation operators

A lot of focus was given to mutation operators, as we believe they are core elements of useful mutation testing tools, albeit they still do not get enough attention in the literature.

Most of the presented research may be also applied to the field of automated refactoring, as the community of automated refactorings seeks applications syntactically 
different than the original one, but having same semantics, i.e., the same what community of mutation testing rejects. Since results from this paper can be used for analysing both equivalence and non-equivalence, one may use them to assess equivalence on refactored application (for a strictly defined set of refactoring operators).

\subsection{Threats to validity}

Since this paper is a theoretical one and presented concepts were not yet implemented, there is a chance that some or all of them will not have expected benefits to actual analysis.

It is possible that in real-world applications independent paths are rare, and, as a result, there will be a little gain due to their analysis. It is also possible that analysis of the mutation path, $x_{1} \cdot x_{2} \cdot \ldots \cdot x_{n}$ will not be efficient enough to detect mutants that were already analyzed and a simple check sum algorithm after mutation will be better in practical approach.

It may also be that reversible mutation operators are virtually useless in real-world programs for a number of reasons - they may either not produce interesting outputs or be hard to create and validate.

Some of commonly used mutation operators cannot be used to form a group -in such cases, only theories applicable to monoids (or even semigroups) may be used. Further, relations between operators may be hard to formulate and their analysis may be inefficient.

While we put effort into making sure that formulas and proofs presented in the paper are correct, there is still a possibility for an error.

This paper also introduces a new threat for mutation testing - it increases difficulty of empirical evaluation of mutation testing strategies. While typically metrics like Mutation Score Indicator $(M S I)$ [32, 34, 33] can be applied, it is not clear how to apply them to an algorithm that removes mutants whose properties can be inferred statically.

\subsection{Further work}

Critical aspect of further work on this subject is to empirically validate theoretical results provided by this paper. Our conjecture is that this formalization may be useful to the reader during a study independent from our own. One of the possibilities we consider particularly attractive is to apply search-based software engineering as a way to filter Higher-Order Mutants before evaluating them against test cases.

Most important theoretical area that needs to be explored in relation to this study is the nature of mutation operators. Describing and analyzing dependencies between various mutation operators, relationship between applying same mutation operator in multiple locations in the source code and researching usefulness of sets of operators forming groups are at the core of further research.

It is also very important to analyze how do classic mutation operators fit into 
presented theory - while in Section 6.3 we did present three ways of transforming them to make them fit, we do not claim that these are the only ones or the best ones. In particular, operator-specific strategies may bring in much benefit. Existing mutation operators - such as statement deletion - are already empirically evaluated and have a proven track of usefulness, therefore it would be beneficial to be able to use them.

To further expand possibility of analysis based on patches and simplify proofs, it may be useful to transform it into some well-known mathematical object, for example a boolean function. How to do this is still a subject of further research.

Performance comparison of different metrics for comparing programs (in order to decide whether further mutations should be applied, or is the application too far from the original one) will bring in great benefit, providing guidance for selection of one of the core parameters of mutation testing system.

Finally, a new metric for evaluating mutation algorithm quality is needed. Using classical metrics like $M S I$ [32, 34, 33] may be biased when the algorithm aims to remove both mutants which will surely be alive and ones which will surely be killed (in unknown proportions). As a result, the numbers will be totally incomparable, and it will not be actually possible to decide whether an algorithm performs better or worse without higher-level evaluation metrics. Perhaps such metrics can be provided by co-evolution of test set and mutation set [8] (one simple example of such metric may be number of additional test cases needed to kill all killable mutants), but this is still subject to be researched.

\section{Conclusions}

Mutation testing is widely known and used for several decades already. Still, a fundamental theory that would make it more efficient and understandable, as well as usable in real-world applications is rather limited. Lack of basic definitions and requirements (such as the nature of mutation operators) makes the area much harder to develop and use in production.

This paper introduces a formal mathematical theory, based on abstract algebra, which can be applied to the mutation testing. It uses a notion of patches, but imposes several new constraints on mutation operators. While no effort in this paper was dedicated to define any set of operators, three approaches were presented to the problem of adjusting existing operators to new constraints. We have also formally defined other items required for a mutation testing tool to be able to apply this theory: a measure of similarity between applications and addressing scheme, which has to be invariant in respect to used operator set.

Then we have presented related theoretical results from patch theory and, as a result, proven that any set of mutation operators for which imposed requirements hold form an inverse monoid. We have also showed an example of how to extract a group from this monoid (imposing additional constraints on mutation operators).

In the end we demonstrated several use cases for the group theory in mutation testing and outlined main directions of further work on the principles of mutation 
testing.

\section{Acknowledgments}

Lech Madeyski was partially supported by the Polish Ministry of Science and Higher Education under Wroclaw University of Science and Technology Grant 0401/0201/18.

\section{References}

[1] Unified Diff Format. https://www.gnu.org/software/diffutils/manual/ html_node/Unified-Format.html. Accessed: 2019-06-21.

[2] Angiuli C., Morehouse E., Licata D. R., and Harper R. Homotopical patch theory. SIGPLAN Not., 49(9):243-256, Aug. 2014.

[3] Arkhangel'skii A. and Pontryagin L., editors. General topology I: basic concepts and constructions dimension theory. Springer, 1990.

[4] Baker R. J. and Habli I. An empirical evaluation of mutation testing for improving the test quality of safety-critical software. IEEE Transactions on Software Engineering, 39(6):787-805, 2013.

[5] Belli F. and Beyazit M. A formal framework for mutation testing. In Proceedings of the 4 th International Conference on Secure Software Integration and Reliability Improvement (SSIRI'2010), pages 121-130, Singapore, Singapore, June 2010.

[6] Bishop D. M. Group theory and chemistry. Courier Corporation, 1993.

[7] Calcagno C., Distefano D., Dubreil J., Gabi D., Hooimeijer P., Luca M., O'Hearn P., Papakonstantinou I., Purbrick J., and Rodriguez D. Moving fast with software verification. In Havelund K., Holzmann G., and Joshi R., editors, NASA Formal Methods, pages 3-11, Cham, 2015. Springer International Publishing.

[8] de Oliveira A. A. L., Camilo-Junior C. G., and Vincenzi A. M. R. A coevolutionary algorithm to automatic test case selection and mutant in mutation testing. In 2013 IEEE Congress on Evolutionary Computation, pages 829-836, Cancun, Mexico, June 2013.

[9] Delamaro M. E., Offutt J., and Ammann P. Designing deletion mutation operators. In 2014 IEEE Seventh International Conference on Software Testing, Verification and Validation, pages 11-20, Cleveland, OH, USA, March 2014.

[10] Delgado-Pérez P., Medina-Bulo I., Domínguez-Jiménez J. J., GarcíaDomínguez A., and Palomo-Lozano F. Class mutation operators for $\mathrm{c}++$ objectoriented systems. annals of telecommunications - annales des télécommunications, 70(3):137-148, Apr 2015. 
[11] DeMillo R., Lipton R. J., and Sayward F. Program mutation: A new approach to program testing. Infotech State of the Art Report, Software Testing, 2:107-126, 1979.

[12] DeMillo R. A., Lipton R. J., and Sayward F. G. Hints on Test Data Selection: Help for the Practicing Programmer. IEEE Computer, 11(4):34-41, 1978.

[13] Deng L., Offutt J., and Li N. Empirical evaluation of the statement deletion mutation operator. In 2013 IEEE Sixth International Conference on Software Testing, Verification and Validation, pages 84-93, Luxembourg, Luxembourg, March 2013.

[14] Estero-Botaro A., Palomo-Lozano F., and Medina-Bulo I. Mutation operators for WS-BPEL 2.0. In 21th International Conference on Software \& Systems Engineering and their Applications, 2008.

[15] Findler R. B. and Felleisen M. Contracts for Higher-order Functions. SIGPLAN Not., 37(9):48-59, Sept. 2002.

[16] Gligoric M., Jagannath V., and Marinov D. MuTMuT: Efficient exploration for mutation testing of multithreaded code. In 2010 Third International Conference on Software Testing, Verification and Validation (ICST), pages 55-64. IEEE, 2010 .

[17] Hamlet R. G. Testing Programs with the Aid of a Compiler. IEEE Transactions on Software Engineering, 3(4):279-290, 1977.

[18] Harman M., Jia Y., and Langdon W. B. A Manifesto for Higher Order Mutation Testing. In Proceedings of the 2010 Third International Conference on Software Testing, Verification, and Validation Workshops, ICSTW '10, pages 80-89, Paris, France, 2010. IEEE Computer Society.

[19] Hu J., Li N., and Offutt J. An analysis of oo mutation operators. In 2011 IEEE Fourth International Conference on Software Testing, Verification and Validation Workshops, pages 334-341, March 2011.

[20] Jacobson J. A formalization of Darcs patch theory using inverse semigroups. ftp://ftp.math.ucla.edu/pub/camreport/cam09-83.pdf, 2009. Accessed: 201806-20.

[21] Jia Y. and Harman M. Higher Order Mutation Testing. Information and Software Technology, 51(10):1379 - 1393, 2009. Source Code Analysis and Manipulation, SCAM 2008.

[22] Johann P. and Ghani N. Foundations for Structured Programming with GADTs. SIGPLAN Not., 43(1):297-308, Jan. 2008.

[23] Johnson S. C. Lint, a C program checker. Technical report, Bell Laboratories, 1978. 
[24] Just R., Ernst M. D., and Fraser G. Using State Infection Conditions to Detect Equivalent Mutants and Speed up Mutation Analysis. CoRR, abs/1303.2784, 2013.

[25] Kim S., Clark J., and McDermid J. The rigorous generation of Java mutation operators using HAZOP. In Proceedings of the 12th International Conference on Software \& Systems Engineering and their Applications (ICSSEA'99), 1999.

[26] Kintis M. and Malevris N. Identifying more equivalent mutants via code similarity. In Proceedings of the 20th Asia-Pacific Software Engineering Conference (APSEC'2013), volume 1, pages 180-188. IEEE, 2013.

[27] Kostrikin A. Introduction into Algebra. Nauka, Moscow, 1977.

[28] Larus J. R. Whole program paths. SIGPLAN Not., 34(5):259-269, May 1999.

[29] Lidl R. and Pilz G. Applied abstract algebra. Springer Science \& Business Media, 2012.

[30] Lyubarskii G. Y. The application of group theory in physics. Elsevier, 2013.

[31] Ma Y.-S., Kwon Y.-R., and Offutt J. Inter-class mutation operators for java. In Proceedings of the 13th International Symposium on Software Reliability Engineering, (ISSRE'2002), pages 352-363, Annapolis, MD, USA, USA, 2002. IEEE.

[32] Madeyski L. Impact of pair programming on thoroughness and fault detection effectiveness of unit test suites. Software Process: Improvement and Practice, 13(3):281-295, 2008.

[33] Madeyski L., Orzeszyna W., Torkar R., and Józala M. Overcoming the Equivalent Mutant Problem: A Systematic Literature Review and a Comparative Experiment of Second Order Mutation. IEEE Transactions on Software Engineering, 40(1):23-42, 12014.

[34] Madeyski L. and Radyk N. Judy-a mutation testing tool for Java. IET Software, $4(1): 32-42,2010$.

[35] Meyers S. and Lejter M. Automatic detection of c++programming errors: Initial thoughts on a lint++. Technical report, Brown University, Providence, RI, USA, 1991.

[36] Mimram S. and Giusto C. D. A categorical theory of patches. CoRR, abs/1311.3903, 2013.

[37] Morell L. A theory of fault-based testing. IEEE Transactions on Software Engineering, 16(8):844-857, Aug 1990.

[38] Morell L. J. A Theory of Error-based Testing. PhD thesis, University of Maryland at College Park, College Park, MD, USA, 1984. 
[39] Navarro G. A guided tour to approximate string matching. ACM computing surveys (CSUR), 33(1):31-88, 2001.

[40] Nguyen Q. V. and Madeyski L. Problems of mutation testing and higher order mutation testing. In van Do T., Thi H. A. L., and Nguyen N. T., editors, Advanced Computational Methods for Knowledge Engineering, pages 157-172. Springer International Publishing, 2014.

[41] Nguyen Q. V. and Madeyski L. Searching for Strongly Subsuming Higher Order Mutants by Applying Multi-objective Optimization Algorithm. In Le Thi H. A., Nguyen N. T., and Do T. V., editors, Advanced Computational Methods for Knowledge Engineering, volume 358 of Advances in Intelligent Systems and Computing, pages 391-402. Springer International Publishing, 2015.

[42] Offutt A. J., Lee A., Rothermel G., Untch R. H., and Zapf C. An experimental determination of sufficient mutant operators. ACM Transactions on Software Engineering and Methodology (TOSEM), 5(2):99-118, 1996.

[43] Offutt A. J. and Pan J. Automatically detecting equivalent mutants and infeasible paths. Software Testing, Verification and Reliability, 7(3):165-192, 1997.

[44] Praphamontripong U. and Offutt J. Applying mutation testing to web applications. In Proceedings of the 3rd International Conference on Software Testing, Verification, and Validation Workshops (ICSTW'2010), pages 132-141, Paris, France, 2010. IEEE.

[45] Runeson P. A survey of unit testing practices. IEEE Software, 23(4):22-29, July 2006.

[46] Schuler D. and Zeller A. Javalanche: efficient mutation testing for java. In Proceedings of the the 7th joint meeting of the European software engineering conference and the ACM SIGSOFT symposium on the foundations of software engineering, pages 297-298, Amsterdam, The Netherlands, 2009. ACM.

[47] Shin D. and Bae D. H. A theoretical framework for understanding mutationbased testing methods. In Proceedings of the IEEE International Conference on Software Testing, Verification and Validation (ICST'2016), pages 299-308, Chicago, IL, USA, April 2016.

[48] Siami Namin A., Andrews J. H., and Murdoch D. J. Sufficient mutation operators for measuring test effectiveness. In Proceedings of the 30th International Conference on Software Engineering (ICSE'2018), pages 351-360, Leipzig, Germany, 2008. ACM.

[49] Smith B. H. and Williams L. An Empirical Evaluation of the MuJava Mutation Operators. In Proceedings of the Testing: Academic and Industrial Conference Practice and Research Techniques (TAICPART-MUTATION'2007), pages 193202, Washington, DC, USA, 2007. IEEE Computer Society. 
[50] Smith J. M. Elemental Design Patterns. Addison-Wesley, 2012.

[51] Sun Y., Wang Q., and Yang Y. FRLink: Improving the recovery of missing issuecommit links by revisiting file relevance. Information and Software Technology, $84: 33-47,2017$.

[52] Tillmann N. and Schulte W. Parameterized unit tests. In Proceedings of the 10th European Software Engineering Conference Held Jointly with 13th ACM SIGSOFT International Symposium on Foundations of Software Engineering, ESEC/FSE-13, pages 253-262, Lisbon, Portugal, 2005. ACM.

[53] Usaola M. P. and Mateo P. R. Mutation testing cost reduction techniques: A survey. IEEE Software, 27(3):80-86, May 2010.

Received 17.01.2019, Accepted 25.10.2019 